and heavy works are risk factors of both KOA and VV. The association of the two diseases is variously assessed in the literature: prevalence of VV in KOA patients varies from 18 to $64 \%$ and prevalence of KOA in VV patients varies from 12 to $68 \%$. This association is still questionable, as no case-controlled study is available.

Objectives To evaluate the possible association of the two diseases in a case-control study.

Methods Three hundred patients with KOA defined according to the ACR criteria (mean age \pm SD: 66 years $\pm 10,64 \%$ women) were age and sex-matched with 300 controls (subjects without any present or past knee pain + normal examination of their knees + normal AP x-rays of their knees on standing extended position: Kellgren-Lawrence stage 0). All classical risk factors were recorded (sports, trauma, heredity, works, meniscectomy, ?). In this epidemiological survey, VV were classified as followed: no VV (V0), simple venous varicosities with dull ache or pressure sensation in the legs which feel heavy after prolonged standing, (V1), or VV with complications (erythema, dermatitis, hyperpigmentation developed along the distal aspect of the leg, or skin ulcers) or which were operated (V2). This grading system was set up assuming that the diagnosis of $\mathrm{VV}$ at stage 1 is sometimes questionable, while $\mathrm{VV}$ at stage 2 is more accurate.

Results There were 95 and 74 V1 patients and 59 and 43 V2 patients in KOA and control groups respectively (Chi2 =9.28; $\mathrm{p}=0.0097)$. This increase of $\mathrm{VV}$ in KOA is also significant in women (chi2 $=9.498 ; \mathrm{p}=0.0087)$, but not in men $(\mathrm{p}=0.34)$. As age, body mass index, hypertension, smoking, sports, carrying heavy loads are confounders in both diseases, a multiple logistic regression was performed. There was a relationship between VV and carrying heavy loads, leading to two separated analyses.

\begin{tabular}{lllll}
\multicolumn{5}{l}{ Abstract SAT0070 Table 1 } \\
& Varicose Veins & RR of Knee OA & Cl 95\% & P \\
\hline Heavy loads (-) & V1 & 1.10 & $0.64-1.87$ & 0.72 \\
Heavy loads (-) & V2 & 1.46 & $0.79-2.70$ & 0.23 \\
Heavy loads (+) & V1 & 2.37 & $1.03-5.45$ & 0.042 \\
Heavy loads (+) & V2 & 3.58 & $0.16-11.04$ & 0.027 \\
\hline
\end{tabular}

Conclusion When there is no carrying of heavy loads, the RR of $\mathrm{KOA}$ is not significantly increased with the presence of $\mathrm{VV}$, while it is when heavy load carriage is also present.

\section{SAT0071 ROFECOXIB SHOWS CONSISTENT EFFICACY IN OA CLINICAL TRIALS, REGARDLESS OF SPECIFIC PATIENT DEMOGRAPHIC AND DISEASE FACTORS}

${ }^{1} \mathrm{LM}$ DeTora, ${ }^{2} \mathrm{D}$ Krupa, ${ }^{2} \mathrm{JA}$ Bolognese, ${ }^{1} \mathrm{RS}$ Sperling, ${ }^{1} \mathrm{EW}$ Ehrich. ${ }^{1}$ Pulmonary-Immunology; ${ }^{2}$ Clinical Biostatistics, Merck Research Laboratories, Rahway, USA

\subsection{6/annrheumdis-2001.446}

\section{Background}

Objectives Analyses were performed to demonstrate the generalizability of the efficacy of rofecoxib (MK-0966, VIOXXTM) in patients with different demographic or baseline disease characteristics, or with $\mathrm{OA}$ in nonsignal joints or multiple locations.

Methods Data were combined for patients with OA of the knee or hip receiving placebo, 12.5 or $25 \mathrm{mg}$ rofecoxib once daily in three 6-week placebo controlled trials, the only trials to date using all 3 treatments. Predefined analyses were performed after categorising patients according to baseline demographics (age, gender, height, weight BMI) and baseline disease characteristics (ARA functional class, joint tenderness, joint stiffness, WOMAC functional subscale, unilateral/bilateral joint involvement, number of joint groups involved). Three primary endpoints: Pain Walking on Flat Surface (WOMAC, 0- $100 \mathrm{~mm}$ VAS), Patient Global Assessment of Response to Therapy (0 to 4 point Likert Scale), and Investigator Global Assessment of Disease Status (0 to 4 point Likert Scale) were analysed. Efficacy treating OA in patients with 1, 2, 3, or 4, joint groups (among interphalangeal/ first carpal-metacarpal joint, spine, hip or knee) affected was analysed for four end points, Patient and Investigator Global Assessments of Response to Therapy and of Disease Status (which provided data on overall aspects of OA, regardless of affected joint). Treatment by factor interactions were tested by ANOVA.

Results Data from 1501 patients were included. For patients taking 12.5 or $25 \mathrm{mg}$ rofecoxib, treatment effects were generally consistent across subgroups categorised by demographic factors: age $(p=0.08)$, gender $(p=0.06)$, race $(p=0.46)$, prior therapy $(\mathrm{p}=0.19)$, weight $(\mathrm{p}=0.10)$, BMI $(\mathrm{p}=0.087)$ or disease characteristics: baseline joint tenderness $(p=0.17)$, WOMAC functional subscale ( $\mathrm{p}=0.52$ ) ARA functional class I, II, or III $(p=0.49)$, duration of OA $(p=0.37)$, or primary study joint $(\mathrm{p}=0.27)$. Greater efficacy was seen in patients with study joint swelling; however, the difference from those without study joint swelling was not clinically meaningful. No significant interactions were observed among patients with unilateral/bilateral involvement or 1, 2, 3, or 4, joint groups affected.

Conclusion No specific factor predicted treatment effect in this study.

This study was funded by a grant from Merck\& Co., Inc.

\section{SAT0072 CELECOXIB DOES NOT AFFECT INDEX JOINT X-RAYS IN OSTEOARTHRITIS (OA) PATIENTS WITH LONG TERM EXPOSURE}

${ }^{1}$ EA Tindall, On Behalf of the 024 Investigators'. ${ }^{1}$ Department of Medicine, Oregon Health Sciences University, Portland; ${ }^{2}$ Research and Development, Pharmacia Corporation, Skokie, USA

\subsection{6/annrheumdis-2001.447}

Background Worsening of joint x-rays has been observed with nonsteroidal anti-inflammatory drug (NSAID) use.

Objectives The effect of celecoxib, a specific cyclooxygenase-2 inhibitor, on index joint (knee or hip) $x$-rays in patients with OA was determined during a long-term safety trial.

Methods X-rays were obtained from all patients prior to entry into a controlled clinical trial, prior to subsequent entry into the long-term safety trial, and at Month 12 or early termination visit of the long-term safety trial. Blinded x-rays were graded on a semiquantitative scale assessing multiple aspects of joint morphology.

Results Overall qualitative interpretation of the individual scores for hip and knee films indicated mild to moderate OA equivalent to an average Kellgren-Lawrence grade of between 2 and 3 at entry to the long-term safety trial. Data from 147 pairs of knee films were analysed prior to and after participation in the longterm safety trial (average duration of treatment, 1 year). Shift table analysis was not significant for any parameters of joint morphology (knee [medial and lateral] joint space narrowing, knee [medial and lateral] spurs, knee [medial and lateral] 